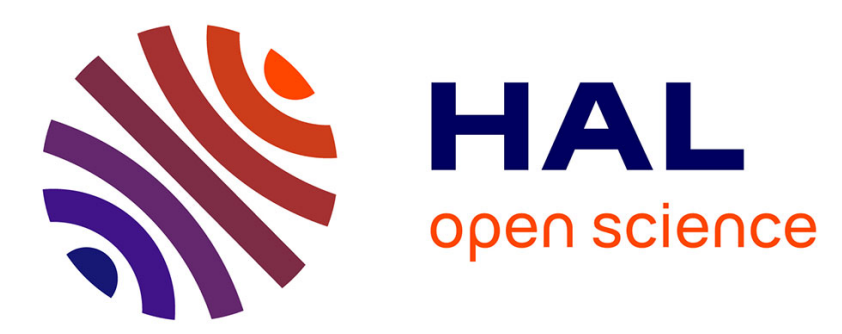

\title{
Noms et corps divins dans les textes bibliques: une approche par le genre
}

Stéphanie Anthonioz

\section{To cite this version:}

Stéphanie Anthonioz. Noms et corps divins dans les textes bibliques: une approche par le genre. Archimède: archéologie et histoire ancienne, 2021, Archimède. Archéologie et histoire ancienne, 8 , pp.32-41. 10.47245/archimede.0008.ds1.03 . halshs-03279599

\section{HAL Id: halshs-03279599 \\ https://shs.hal.science/halshs-03279599}

Submitted on 6 Jul 2021

HAL is a multi-disciplinary open access archive for the deposit and dissemination of scientific research documents, whether they are published or not. The documents may come from teaching and research institutions in France or abroad, or from public or private research centers.
L'archive ouverte pluridisciplinaire HAL, est destinée au dépôt et à la diffusion de documents scientifiques de niveau recherche, publiés ou non, émanant des établissements d'enseignement et de recherche français ou étrangers, des laboratoires publics ou privés. 
DOSSIER THÉMATIQUE 1

SIUE DEUS SIUE DEA. DÉNOMINATIONS DIVINES DANS LES MONDES GREC ET SÉMITIQUE : UNE APPROCHE PAR LE GENRE

1 Corinne Bonnet, Thomas Galoppin, Adeline Grand-CLÉment

Que fait le genre aux dénominations divines, entre mondes grecs et sémitiques?

17 Stella GEORGOUDI

Qu'est-ce qu'une « Grande » (Megalê) divinité en Grèce ancienne ?

32 Stéphanie ANTHONIOZ

Noms et corps divins dans les textes bibliques : une approche par le genre

42 Audrey VASSELIN

De la bonne manière de nommer Athéna à Athènes :

I'emploi de hê theos dans les inscriptions athéniennes (VIe-IVe siècles av. J.-C.)

\section{Aleksandra KUBIAK-SCHNEIDER}

Women in the votive dedications from Palmyra

65 Marie Augier

Dénominations divines, genre des acteurs du rituel et des agents cultuels : le cas des prêtrises de Dionysos

84 Adriano ORSINGHER

Giving a face to a name. Phoenician and Punic Divine Iconographies, Names and Gender

98 DOSSIER THÉMATIQUE 2

TRADITION ET TRANSMISSION DANS L'ANTIQUITÉ : RÉFLEXIONS INTERDISCIPLINAIRES

159 ACTUALITÉ DE LA RECHERCHE

QUOI DE NEUF À L'OUEST DE STRASBOURG ? KOENIGSHOFFEN :

ÉTAT DES LIEUX ET DÉCOUVERTES RÉCENTES

195 VARIA 


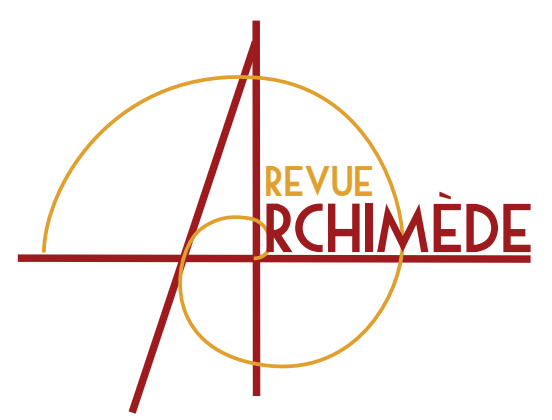

ARCHÉOLOGIE ET HISTOIRE ANCIENNE

\section{NOMS ET CORPS DIVINS DANS LES TEXTES BIBLIQUES : UNE APPROCHE PAR LE GENRE}

\section{Stéphanie ANTHONIOZ}

UMR 8167 Orient et Méditerranée («Mondes sémitiques»)

Stephanie.anthonioz@orange.fr

\section{RÉSUMÉ}

La contribution qui suit propose d'explorer, dans les textes bibliques, les attributions de « genre » appliquées à la représentation du divin. Deux aspects sont pris en considération, à travers quelques cas précis, les dénominations divines, d'une part, et les représentations du corps divin, d'autre part. Cette contribution éclaire la fluidité des représentations divines au Levant, c'est-à-dire leurs variables ainsi que leurs constantes, pour lesquelles les textes bibliques, en regard des sources épigraphiques et matérielles, sont un témoin précieux.
If the question of divine representation in Antiquity is a complex matter, can the notion of gender clarify the intention at work in representing any particular god? The following contribution aims at exploring biblical texts with the interpretative tools of gender. Two dimensions are taken into consideration, divine denominations and divine bodies. This contribution highlights the fluidity of ancient representations of the divine with regard to gender and biblical texts appear as precious witnesses along epigraphical and material sources.

\section{KEYWORDS}

Gender, biblical texts, Astarte ('aštōret), Queen of Heaven, Šadday, Ašerah, Anat-Bethel, Seraphim, Cherubim, Lady Wisdom. 
Que les dieux aient un corps à l'image de I'humain - ce que I'on nomme anthropomorphisme - constitue une représentation possible parmi d'autres, astrales, animales, végétales ou encore minérales. La représentation et même la matérialité de l'objet ou plutôt du sujet divin n'ont pas empêché les Anciens de penser immanence et transcendance. Ces représentations sont diversement documentées au Levant. Il suffit de citer ici deux contributions au titre évocateur, The Many Faces of the Goddess et « The Many Faces of God: Divine Images and Symbols in Ancient Near Eastern Religions [1] ». Cette diversité et cette fluidité sont aujourd'hui de plus en plus reconnues dans les études bibliques, comme le montrent les travaux de Benjamin D. Sommer ou Izaak J. de Hulster [2]. Il faut, par ailleurs, garder à l'esprit que la pensée sémitique ne distingue pas le corps de la personne : la personne est saisie comme l'assemblage de ses parties, à savoir le corps, mais aussi ses noms, ses fonctions et ses images [3]. Aussi le corps fait-il partie de la définition de l'être. Il n'y a aucune séparation entre physique et psychique au point que certaines parties corporelles sont responsables de certaines fonctions cognitives et peuvent représenter symboliquement la personne [4]. Or cette conception s'applique au monde divin, tout en s'élargissant : les dieux sont présents et agissent à travers leurs représentations cultuelles, leurs lieux, leurs attributs. Aussi la notion de corps divin, comme celle des dénominations ou des représentations divines, doit être saisie dans une diversité qui s'accompagne d'une grande fluidité, avec à la fois des constantes et des évolutions. Au regard de toute représentation, et avant d'aborder la question du genre, il convient ainsi de souligner les choix linguistiques et iconographiques, mais aussi fonctionnels pour désigner telle ou telle divinité. Une

[1] Cornelius 2004 ; CORnelius 1997, p. 21-43.

[2] HULSTER 2017 ; SOMMER 2009. Il faut aussi mentionner ceux de Benedikt Hensel qui renouvellent considérablement le débat historique et exégétique en insistant sur la diversité des yahwismes aux différentes époques. Hensel 2020.

[3] Pongratz-Leisten 2011, p. 137-187. Voir également WeSTENHOLZ 2012, p. 459-477. divinité est ce qu'elle est dans la diversité de ses noms et épithètes, attributs et représentations, certes, mais dans la limite aussi de ces derniers. Cette particularité qu'implique la « réalisation » de toute représentation appartient de fait à l'image comme au langage. Faut-il dire alors combien la question du genre appartient au monde de la représentation, image ou langage, et donc à l'intention ou à la motivation de « I'œuvre » réalisée ?

Ces remarques introductives permettent $d^{\prime}$ insister sur la diversité des modes de représentation et de souligner leur coexistence dans le monde ancien. Elles permettent aussi de relativiser les choix de représentations, lorsqu'il s'agit de penser le divin. Ce constat amène alors à intégrer à la réflexion la notion de «genre » et à se demander ce qui est en jeu lorsque I'on recourt à une formulation féminine plutôt que masculine. Par exemple en akkadien, le dieu Šamaš, dieu Soleil, en tant que puissance divine, présente des similitudes avec les autres divinités solaires du Proche-Orient ancien, souvent désignées dans les textes cunéiformes par le logogramme UTU : le recours au logogramme d'origine sumérienne, non genré grammaticalement, permet de désigner une même entité divine - ici en l'occurrence l'astre solaire - qui sera lue différemment selon les langues. Or, dans les pays ouest-sémitiques, les divinités solaires sont plutôt féminines. À Ougarit, la divinité solaire est la déesse Šapaš, dont le nom est formé à partir de la même racine que celui de Šamaš. On peut penser aussi à la déesse Arinna au sommet du panthéon officiel hittite. Pourquoi dire le soleil divinisé par le féminin plutôt que par le masculin ? Y a-t-il seulement un enjeu à telle ou telle attribution de genre à une représentation divine ? Et les représentations sontelles toujours aussi tranchées?

[4] Les reins sont, par exemple, le lieu du discernement, le cœur mais aussi les oreilles, celui de l'intelligence. Les yeux dans leur transparence peuvent être associés, par contre, à différents sentiments. Les narines peuvent signifier la colère comme la langue ou la bouche, la calomnie. Les mains, les bras et les jambes désignent souvent la force ou la puissance. Voir l'ouvrage séminal DHORME 1923. 
La contribution qui suit propose d'explorer les attributions de « genre » appliquées à la représentation du divin dans les textes bibliques. Deux aspects sont pris en considération à travers quelques exemples précis, les dénominations divines, d'une part, et les représentations du corps divin, d'autre part. Cette contribution éclaire la fluidité des représentations divines au Levant, et les textes bibliques, en regard des sources épigraphiques et matérielles, apparaissent comme un témoin précieux.

\section{PENSER LE GENRE DES DÉNOMINATIONS DIVINES DANS LA BIBLE HÉBRAIIQQUE}

Pour réfléchir au genre des dénominations divines, dans les textes bibliques, je voudrais présenter quelques cas ('aštōret, la Reine du ciel, Šadday) qui permettent de mettre en perspective la question du genre et ses difficultés. Cette mise en perspective permettra, en deuxième partie, d'aborder la notion de corps divin.

\section{IŠTAR SÉMITIQUE ET 'AŠTŌRET BIBLIQUE}

La question du genre se pose d'abord au sujet des dénominations divines. Nous l'avons entrevu à propos de Šamaš / Šapaš. Ainsi, un nom comme Ištar d'origine sémitique ('ttr), peut-être d'une racine signifiant la richesse [5] ou encore l'étoile du matin [6], est attesté à la fois comme nom d'une divinité masculine dans les sources sud-arabiques et à Ougarit [7], et comme nom d'une divinité féminine, dans les sources sud-arabiques également ('ttrm) [8] mais plus fréquemment dans les sources ouest-sémitiques ('ttrt) [9] - à Ougarit [10], en Phénicie [11] et dans les textes bibliques (ערתר, 'aštōret, 1 R 11,5.33 ; 2 R 23,13). La dénomination masculine - c'est-à-dire sans marque grammaticale du féminin - pourrait rendre compte d'une divinité à l'origine androgyne, la forme masculine renvoyant à Vénus étoile du matin, la forme féminine à l'étoile du soir [12]. La forme de féminin pluriel fonctionne comme générique - les déesses - dans les sources néo-assyriennes (ištarātu) comme dans les sources bibliques (עשתרת, 'aštārôt, Jg 2,13;10,6 ; 1 $\mathrm{S} 7,3 ; 12,10 ; 31,10)$, même si dans ce dernier cas on peut montrer que, plus qu'un usage générique, il s'agit d'un usage polémique, plus spécifiquement d'un terminus technicus dans la polémique contre I'idolâtrie [13]. Le nom d'Ištar est intéressant : si le caractère androgyne de la déesse est démontré depuis longtemps, il est notoire que les différents témoins, au Levant tout du moins, ont en général eu recours à une finale féminine pour l'identifier. Dans ce cas, le caractère féminin de la divinité est explicité. Sans doute faut-il mettre en rapport cette sexuation de la divinité avec ses différentes fonctions (fécondité, vitalité), mais cela ne peut être prouvé. Il est notable que, dans la critique biblique et la polémique contre l'idolâtrie, ce caractère genré de la divinité est explicitement mis au service de l'idéologie yahwiste : non seulement c'est une divinité étrangère qui est dénoncée, en regard de la divinité masculine et dynastique Yhwh, mais, en plus, elle est féminine.

\section{LA REINE DU CIEL (MELEKET HAŠŠĀ- MAYIM)}

Si I'on poursuit I'analyse de la polémique et de la critique biblique, il faut s'arrêter sur le titre « Reine du ciel » qui est employé à quelques reprises dans le livre de Jérémie :

${ }^{17} \mathrm{Ne}$ vois-tu pas ce qu'ils font dans les villes de Juda et dans les rues de Jérusalem : ${ }^{18}$ les enfants ramassent du bois (הבנים מלקטים עצים), les pères allument le feu (והאבות מבערים את האשים (והשים ) et les femmes pétrissent la pâte (והנשים לשות בצקר) pour faire des gâteaux à la Reine du ciel (לעשות כונים למלכת השמים).

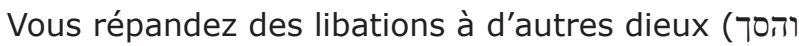
נסכים לאלהים אחרים), et ainsi vous m'offensez. (Jr 7,17-18)

Le cadre de cette référence met en exergue la dimension rituelle, judéenne et surtout familiale du culte rendu à la Reine du ciel. Cette dimension est confirmée plus loin :
[5] MÜLLER 2001, p. 423-434.

[6] L'étymologie serait celle d'un substantif emprunté à l'indo-européen « étoile », ayant généré les différentes formes attestées dans les langues sémitiques. Voir WILSONWRIGHT 2016, p. 12 ; BLAŽEK 1996, p. 133-141.

[7] SмIтн 1995, p. 627-640.

[8] BRON 1999-2000, p. 437.

[9] À l'exception de la ville d'Ebla où le nom est noté ${ }^{d} A s ̌$-tár [10] BORDREUIL 1998, p. 545-547.
[11] Sur le sarcophage de Tabnit à Sidon daté du VIe s. (KAI 13), le roi est « prêtre de 'štrt »; sur celui de Eshmounazor, son fils (KAI 14), sa mère est présentée comme «prêtresse de 'štrt ». La divinité est bien attestée en Égypte et dans l'inscription de Deir 'Alla où elle apparaît sous la forme ammonite Šagar-et-'Aštar. Pour l'ensemble du dossier documentaire, voir BONNET 1996.

[12] ARCHI 1993, p. 71-78.

[13] ANTHONIOZ 2014, p. 125-139. 
${ }^{17}$ Nous allons faire tout ce que nous avons dit : brûler des offrandes à la Reine du ciel (לקטר למלכת השמים), lui verser des libations (והסיך לה נסכים), comme nous I'avons fait dans les villes de Juda et dans les rues de Jérusalem, nous-mêmes, nos pères, nos rois, nos ministres, alors nous avions du pain à satiété et nous vivions heureux sans connaître de malheur. ${ }^{18}$ Depuis que nous avons cessé de brûler des offrandes à la Reine du ciel et de lui verser des libations, nous manquons de tout et nous périssons par l'épée et par la famine. ${ }^{19}$ Et quand nous, nous brûlons des offrandes à la Reine du ciel et que nous lui versons des libations, est-ce sans la collaboration de nos maris que nous lui préparons des gâteaux qui la représentent (אמבלעדי) (אנשים עשינו לה כונים להעצבה (ניה (-17), et que nous lui versons des libations ? (Jr 44,17-19).

Cette seconde référence plus longue développe l'aspect technique et social du culte : outre les offrandes, des libations sont versées ; outre le cadre familial, les autorités officielles sont concernées. La forme du gâteau semble de quelque importance [14] et pourrait renvoyer à une représentation divine. Comme l'a souligné Mathias Delcor, il faut noter la mauvaise humeur du Massorète qui vocalise meleket et non malkat [15] ! Bien évidemment ces références sont à intégrer à un système de polémique et de critique religieuse. Mais, mis à part le titre, les références ne permettent pas de documenter le caractère céleste de la divinité en question. Il est clair seulement qu'elle joue un rôle de premier plan dans les questions de fertilité et d'abondance mais de protection aussi contre les malheurs et la guerre. Or les gâteaux kawwānîm rappellent un rituel lié à la divinité Ištar dont le titre de Reine du ciel (šarrat šamē) est non seulement attesté dans les sources, mais traduit son nom sumérien : [N]IN.AN.NA[K]. L'hébreu kawwān serait ainsi à rapprocher de la forme akkadienne kawânu / kamânu, le substantif kamānu désignant un gâteau sucré préparé avec des figues et du miel (selon le CAD), nourriture de berger et offrande en particulier à la divinité Ištar. Aussi s'agirait-il de la même divinité selon M. Delcor. Cependant l'auteur admet qu'Ištar n'est pas seule Reine du ciel ; Astarté l'est aussi et les caractéristiques de cette dernière sont amplement documentées en Égypte comme en Phénicie au Ier millénaire [16]. Susan Ackerman défend alors une solution syncrétiste, la Reine du ciel partagerait des traits de I'Astarté ouest-sémitique et des traits de I'Ištar est-sémitique [17]. Sans doute faut-il simplement renoncer à rechercher derrière la dénomination une autre identité que celle qui est visée dans une dimension ici manifestement judéenne et communautaire [18]. Quoiqu'il en soit, et pour notre propos, le titre de Reine du ciel est à la fois témoin et vestige d'une divinité féminine céleste. Il témoigne également d'une entité souveraine en regard de la divinité Yhwh. Cependant, dans la perspective polémique du texte, la dimension féminine, accentuée par la forme meleket, est encore mise en exergue par le rôle cultuel marqué des femmes. Ce rôle est ridiculisé, mais ironie du texte, ce n'est pas sans l'accord de leur mari que ces femmes vouent un culte à la divinité en question. La dimension féminine est exploitée en vue d'une idéologie critique et polémique et reflète ainsi à la fois des enjeux religieux, l'exclusivisme yahwiste, mais aussi sociaux (la place de la femme). La féminité de la déesse est ici construite par l'intermédiaire du genre grammatical et du genre des acteurs humains, ne fournissant qu'une vue partielle et partiale sur la puissance divine en question et son culte.

\section{ŠADDAY: ANCIENNE ÉPITHÈTE FÉMININE ?}

Un dernier cas est plus difficile à analyser : celui du nom biblique aujourd'hui assimilé à une épithète $\mathrm{d}^{\prime} \mathrm{El}$, Šadday (שדי). Comme le rappelle Harriet Lutzky [19], on considère depuis longtemps que cette dénomination divine est à rapprocher de la racine sémitique qui signifie «poitrine » (שT) [20]. Le suffixe archaïque féminin -ay, attesté en ouest-sémitique, amorrite et ougaritique, l'est surtout dans les noms d'entités divines ou mythologiques. Aussi trouve-t-on à Ougarit les noms des divinités féminines Tallay (țly bt $r b$, « fille de la rosée »), Arsay (arșy bt y'bdr, « fille de y'bdr ») et Pidray (pdry bt $a r$, « fille de la lumière »), que I'on explique comme « celle du phénomène naturel (dont il est question) » [21]. Selon cette construction, Raḥmay serait ainsi « celle de l'utérus » et Šadday, « celle de la poitrine ». Il est notable que le seul nom biblique, outre

[14] VRIEZEN 2001, p. 69-71; VRIEZEn 1996, p. 251-264.

[15] DELCOR 1982, p. 103.

[16] BONNET 1996 ; OLYAN 1987, p. 161-174.

[17] Voir ainsi ACKERMAN 1999, p. 21-32.

[18] Ainsi ZerneCKe 2013, p. 227-242.

[19] LUTZKY 1998, p. 15-36.

[20] Au sujet de l'étymologie du nom et des différentes références littéraires, voir en dernier lieu WILSON-WRIGHT 2019, p. 149-166. L'auteur, comme le titre l'indique, propose

une nouvelle étymologie du nom, fondée sur la racine « venir en aide ». Les références bibliques se trouvent en Gn 17,1 ; 28,$3 ; 35,11 ; 43,14 ; 48,3 ; 49,25$; Ex 6,3 ; Nb 2,12 ; $10,19.25 ; 24,4.16$; Rt 1,20 ; Jb 5,$17 ; 6,4.14 ; 8,3.5$; 11,$7 ; 13,3 ; 15,25 ; 21,15.20 ; 22,17.23 .25 ; 27,10 ; 29,5$; $31,2.35 ; 32,8 ; 33,4 ; 37,23 ; 40,2$; Ps 22,$10 ; 68,15$; 91,$1 ;$ Ct 1,$13 ; 8,1 ;$ Ez 1,$24 ; 10,5 ; 23,21$.

[21] Noter que les traductions sont débattues particulièrement pour Pidray et Arsay. Voir Wiggins 2003, p. 83-101. 
Šadday, à présenter un tel suffixe soit le nom de Saray : peut-être le passage au nom Sarah atteste-t-il qu'à un moment la finale -ay n'était plus perçue comme un féminin [22] ? L'hypothèse d'une divinité Šadday, liée elle aussi à la fertilité, a été développée dans une contribution de Maurice Canney [23]. Ainsi, la divinité ancienne, hébraïque ou cananéenne, aurait été une divinité-mère, secondairement transformée en dieu. Cette hypothèse est parfois considérée [24], plus souvent rejetée. Mais H. Lutzky poursuit l'analyse en s'appuyant sur les sources ougaritiques, puisque Ašerah / Atirat est certes « génitrice de ses enfants / les dieux » mais également rahmay (litt. « celle de l'utérus »), donc métaphoriquement pleine de compassion [25]. Appelée comme Anat «nourrice des dieux », Ašerah nourrit dieux et mortels, elle les protège. Elle est donc dea genetrix et dea nutrix. Or il est notable que, dans les sources d'Ougarit, on recourt à l'épithète rahmay-genetrix mais à aucune épithète nutrix [26]. L'hypothèse proposée est ainsi que šadday aurait précisément été cette épithète :

«Rahmay and Shadday, Genetrix and Nutrix, would then be parallel epithets. Shadday read as a feminine form would necessarily be early West Semitic, as Asherah is. The epithets Rahmay and Shadday may have parted company long before the period in which the Ugaritic myths were recorded. But though not found in the same place, the two epithets date to roughly the same period. Both the divine name Shadday and the Hebrew theophoric names with the element Shadday found in the Priestly genealogies (Num. i) are considered pre-Mosaic; the theophoric element Shadday has also been found in a Canaanite name from a $14^{\text {th }}-13^{\text {th }}$ century BCE Egyptian context. 》 [27]

L'hypothèse se verrait confirmée par le passage poétique de la bénédiction de Jacob, avec les références à El et Šadday, c'est-à-dire aux bénédictions de la poitrine et de l'utérus (ברכת שדים ורחם, Gn 49,25). Selon certains, le verset contiendrait une référence explicite au père $\mathrm{EI}$ mais implicite à la mère Ašerah, par ses épithètes šadday et rahmay ou nutrix et genetrix. De manière intéressante, les versions grecque et samaritaine du verset 26 pourraient corroborer I'hypothèse du couple divin, puisqu'on y trouve l'expression « bénédictions du père et de la

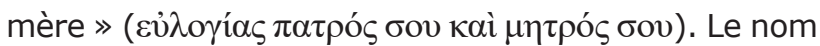
composé El Šadday offre-t-il la possibilité de penser comme originelle I'association du divin El et de sa parèdre Šadday, une association peut-être cultuelle ? Ces évolutions ne sont pas rares dans les sources: d'une part, la divinisation d'une épithète et, d'autre part, la transformation de deux noms étroitement associés qui finissent par désigner une entité nouvelle comprise dans l'unité sinon I'union de deux [28]. N'est-ce pas précisément le cas d'Anat-Yahu dans les sources d'Éléphantine [29] ?

Ces trois cas de dénomination divine et féminine (?) permettent de clarifier la notion de genre selon ses deux acceptions : le genre grammatical et la représentation sociale du genre. Dans le cas de la Bible hébraïque, la dimension polémique paraît étroitement liée à la représentation du féminin, auquel cas la notion de genre convoque les représentations sociales de la Judée au $\mathrm{I}^{\text {er }}$ millénaire av. n. è. Pour autant la dimension religieuse ne peut être occultée, puisque cette polémique n'existe que dans le cadre d'une évolution théologique et d'une idéologie de Yhwh seul, ce que l'on a appelé exclusivisme yahwiste. Mais la nature, féminine ou non, de ces divinités, dans le cadre des cultes qui leur sont rendus et dans le cadre de leurs représentations matérielles, reste étonnamment peu documentée par les textes bibliques, qui s'emparent donc du féminin dans une intention exclusivement critique et polémique. La notion de genre permet ainsi de mettre en avant la manière dont les textes bibliques construisent les dieux des autres dans la perspective yahwiste, en jouant sur les seuls aspects « féminins » de certaines dénominations ('aštōret), ou encore de certaines dénominations associées aux acteurs du culte (Reine du ciel), enfin l'aspect féminin absorbé dans un nom double (El Šadday). Le genre des dénominations divines devient donc un enjeu, puisque manifestement ces textes sont yahwistes, au sens d'une idéologie au service de Yhwh seul. Il s'agit maintenant de se détourner des dénominations pour appréhender la représentation de Yhwh de manière plus large, à travers son corps, et par cette dimension du corps définie plus haut, les parèdres, les attributs et les objets symboliques de la divinité Yhwh.
[22] LUTZKY 1998, p. 17-18.

[23] CANNEY 1922, p. 332.

[24] Selon Biale 1982, p. 240-256, El Šadday aurait été une divinité androgyne de la fertilité incorporant l'image d'Ašerah, cohérente avec le « monothéisme androgyne de Gn 1 ». L'image de la poitrine a une connotation religieuse importante puisqu'elle symbolise à la fois la transmission et la protection divine.

[25] LUTZKY 1998, p. 20-21.

[26] Tanit est, en effet, attestée comme divinité nourricière. BONNET \& NIEHR 2014, p. 94-95.

[27] LUTZKY 1998, p. 21.

[28] LUTZKY 1998, p. 32

[29] VAN DER TOORN 1992, p. 80-101. 


\section{PENSER LE CORPS DIVIN DANS LA BIBLE HÉBRAIIQUE}

Comme on I'a souligné en introduction, on ne peut plus aujourd'hui penser le corps divin sous une forme matérielle unique. Je voudrais, dans le cadre de cette contribution, intégrer à la notion du corps de Yhwh les représentations proches de la divinité, qui témoignent d'une nature partagée. J'intègre donc à la réflexion sur le genre les parèdres, certaines images matérielles ou animales qui re-présentent manifestement la divinité. Le but est toujours de chercher à savoir si la notion de genre permet de mieux comprendre les représentations divines et les intentions à l'œuvre dans ces représentations. Quatre cas sont évalués : Yhwh et son Ašerah, Bethel et Anat-Bethel, Séraphins et Chérubins, enfin, Dame Sagesse.

\section{«YHWH ET SON AŠERAH »}

Ašerah est le nom d'une divinité féminine dont l'étymologie est communément rattachée à la racine nord-ouest-sémitique ${ }^{*}$ 'tr, qui signifie « suivre » au sens d'emboîter le pas. Cette étymologie permet d'expliquer le rôle en général de parèdre auprès de la divinité principale [30]. Atirat est célèbre dans les sources ougaritiques où elle est « mère / génitrice des dieux » (qnyt $\mathrm{ilm}$ ) et sage-femme des rois. Son épithète principale la rattache au monde de la mer ('trt ym) [31]. Atratu est aussi attestée à Mari ( ${ }^{d} A s^{-}$ ra-tum $/{ }^{d} A$-ši-ra-tum) comme parèdre du dieu Amurru, mais cette fois elle est rattachée au monde des steppes et des montagnes comme son époux. Aussi est-elle la parèdre de Baal ou Yhwh dans certaines sources bibliques et sans doute épigraphiques [32]. Ašerah est célèbre, si I'on peut dire, dans la Bible hébraïque : si elle est attestée comme divinité, c'est toujours - on I'a compris - au cœur d'une polémique, de sorte que ni son identité ni son culte ne sont documentés précisément. Cette difficulté est compliquée par le recours fréquent à une forme de pluriel, Ašerîm. Et la relation entre les deux entités est hautement débattue [33]. Faite de bois $(\mathrm{Jg} 6,26)$ que I'on coupe (Ex 34,$13 ; \mathrm{Jg}$ 6,25 ) et que l'on brûle (Dt 12,3; 2 R 23,6.15) ou bien arbre vivant que I'on plante (Dt 16,21 ) et que I'on

[30] Hess 1996, p. 209-219 ; Margalit 1990, p. 264-297. [31] Cross 1973, p. 67. Mais du « jour » selon BINGER 1995, p. 3-18.

[32] Voir les célèbres inscriptions de Kuntillet 'Ajrud et Khirbet el-Qom avec l'attestation «Yhwh et son Ašerah ». Voir encore les références bibliques qui font de la divinité la parèdre de Baal mais aussi de Yhwh (1 R 18,19; 2

R 13,6). La bibliographie est immense sur le sujet, par exemple, MARgalit 1990, p. 284 ; Thomas 2016, p. 121 arrache (Mi 5,13; 2 R 23,14), l'Ašerah est le plus souvent un objet de bois que I'on fabrique ( $1 \mathrm{R} 14,15$; $16,33 ; 2 \mathrm{R} 17,16 ; 21,3$; Is 17,8$)$, que I'on érige comme les mașșebôt $(2 \mathrm{R} 17,10)$ et qui reste dressé ( 2 R 13,6 ; Is 27,9 ) ce qui évoque l'idée d'un poteau ou d'un pieu. Une position prudente - Ašerah est divinité et symbole - est en général adoptée [34], qui est cohérente avec une notion de divinité qui embrasse le nom, la puissance, et les représentations. Les auteurs considèrent parfois une évolution historique : le caractère divin de la représentation se serait perdu au profit de l'objet seul. Mais, de notre point de vue, il ne s'agit pas d'une évolution mais plutôt d'une stratégie qui vise à réduire à rien les autres dieux et leurs représentations, toujours dans le cadre de la polémique religieuse. On notera que la représentation, végétalisée, n'est pas à nouveau particulièrement genrée, sinon par les références qui renvoient à la divinité féminine Ašerah. L'attribution de genre relève donc, comme pour Astarté, d'un système biblique polémique qui range «l'Ašerah » comme objet en référence évidente à une divinité « féminine » et « étrangère », en opposition flagrante avec la divinité jalouse et exclusive Yhwh.

\section{LE CAS DE BETHEL ET ANAT-BETHEL}

Mieux que les bétyles ou mașșebôt, soumis à la même polémique que les ašerîm, il semble que le cas des divinités Bethel et Anat-Bethel soit particulièrement éclairant pour notre propos. Ici je considère la parèdre divine comme étroitement associée au corps divin. Bethel et Anat-Bethel sont deux divinités attestées dans les lieux de culte du royaume du Bit Agusi dont la capitale araméenne est Arpad [35]. Selon H. Niehr, le dieu Bethel est une pierre sainte divinisée et vénérée comme divinité à part entière : le lieu de culte pourrait être Bet Laha, à environ 30 km à l'ouest d'Alep [36]. Le dieu Bethel et sa parèdre Anat-Bethel sont documentés par le traité entre le roi Assarhaddon (680-669) et le roi Ba'al de Tyr, vers 675 / 674 av. n. è., dans la liste des dieux du serment (SAA II no 5 VI 6). Ils sont aussi énumérés dans la liste des dieux du serment, cités dans le traité de succession du roi Assarhaddon (SAA II no 6, 467 § 54A), vers 672. Les dieux Bethel

191 ; THOMAs 2017, p. 157-218.

[33] Pour une approche prudente, voir Richelle 2018, p. 95-119.

[34] Par exemple, PETTEY 1990 ; DAY 1986, p. 408.

[35] BONNET \& NiEHR 2014, p. 274-275.

[36] Ce lieu pourrait être identique au lieu appelé Bethel, cité dans les traités de Sfiré (KAI 224,34). Voir BONNET \& NiEHR 2014, p. 280 
et Anat-Bethel auraient, selon I'auteur, été installés à Samarie dans une relation étroite avec le dieu du pays, Yhwh. Bethel est assimilé à Yhwh, tandis qu'AnatBethel prend, de son côté, les traits d'Anat-Yahu, et devient parèdre du dieu Yhwh. La transposition littéraire de ce processus se rencontre, non pas dans les textes bibliques, mais dans les papyrus d'Éléphantine du Ve s. [37], issus de la communauté judéo-araméenne de la diaspora. Cet exemple permet manifestement de vérifier que la fluidité est au cœur du monde divin, une fluidité aussi dans I'association des divinités et leur réception. Elle permet pour notre propos de mettre en avant un autre point important qui ressort de l'analyse, I'association étroite entre masculin et féminin et le caractère partial des textes bibliques qui, dans leur transmission même, ont fait disparaître bien des aspects féminins du divin, sinon de la religion.

\section{LA GARDE RAPPROCHÉE DU CORPS DIVIN : SÉRAPHINS ET CHÉRUBINS}

Les visions divines sont un fait rare dans les textes bibliques, ce qui a quelque chance de refléter déjà l'interdit deutéronomique de toute représentation divine. Pourtant quelques visions prophétiques trouvent place dans un cadre souvent cultuel, que I'on songe à Isaïe ou Ézéchiel, et laissent apparaître une représentation anthropomorphique de la divinité, plutôt cosmique et royale en Isaïe, d'apparence humaine en Ézéchiel (Ez 1,26). Au sein de ces deux visions, des formes animales sont présentes, serāpîm pour la première et kerubîm pour la seconde. Si l'on garde à l'esprit le rapport entre la divinité et ses attributs ou symboles, il est alors notable pour notre propos de souligner I'intention à l'œuvre dans la conception des kerubîm particulièrement. La vision inaugurale du livre d'Ézéchiel est construite de manière érudite et structurée. La structure implique une révélation graduée qui part d'éléments naturels pour aboutir à une humanité d'Adam en passant par quatre créatures aussi divines qu'elles sont humaines et animales [38]. Ce sont ces créatures qui méritent l'attention du détail. Elles sont caractérisées anthropomorphiquement certes, avec les références à la « ressemblance d'un homme » $(1,5)$, aux « mains d'hommes » $(1,8)$ et à la face d'homme $(1,10)$, mais la rhétorique ainsi que la présence des ailes puis des faces animales [39] comme auparavant celle des pieds de veau mettent à distance cette humanité. Par ailleurs, la symbolique du feu et son omniprésence et récurrence parmi les éléments théophaniques soulignent la transcendance de l'objet (1,4-5.13-14). La question du genre est alors d'autant plus importante que les êtres vivants sont tantôt traités grammaticalement comme des sujets féminins (להנה,

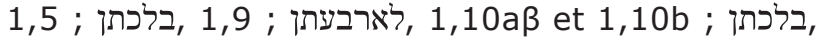

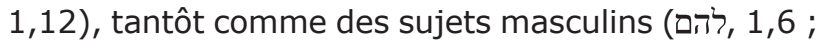
, 1,12a et b). De plus, la structure rythmique associe ces masculins et ces féminins grammaticaux, de sorte qu'on ne peut dire précisément si ces entités divines sont masculines ou féminines. C'est donc un autre aspect du divin qui est mis en lumière, non pas l'indifférenciation, non pas l'association des genres, mais quelque chose qui relève de la complémentarité des genres et semble refléter, d'une autre manière, l'indicible divin dans la diversité de ses représentations. Ce cas reste étonnant au vu de l'idéologie yahwiste exclusive que servent les textes bibliques et que l'analyse ici n'a de cesse de souligner. Mais il est certainement à mettre en relation avec les différents outils littéraires du livre qui permettent de construire une divinité Yhwh à la fois cosmique et transcendant le cosmos [40].

\section{DAME SAGESSE}

Un dernier cas, enfin, pour notre étude est celui de Dame Sagesse, puisque de manière unique le féminin est réintroduit au côté de Yhwh à une époque plus tardive, hellénistique. Les textes bibliques concernés sont peu nombreux ( $\operatorname{Pr} 1-9 ; 31$; Siracide ; livre de la Sagesse ou Sophia Salômônis). Ainsi, entre la première collection des Proverbes, que I'on peut dater vers le III ${ }^{\mathrm{e}}$ siècle av. $\mathrm{n}$. è., et le livre de la Sagesse au I $^{\text {er }}$ siècle de notre ère, on tient en quelque sorte le cadre temporel et spatial - de la Judée à Alexandrie du développement de la figure de la sagesse personnifiée. Les autres témoins s'inscrivent dans cet
[37] VAN DER TOORN 2016, p. 668-680; VAN DER TOORN 1992 , p. $80-101$

[38] La théophanie $(1,4)$ est suivie par la description des quatre créatures vivantes (1,5-14, plus tard identifiées aux chérubins), la / les roue(s) $(1,15-18)$, les quatre et les roues (1,19-21), l'étendue / firmament et la voix de Šadday $(1,22-25)$, le trône, I'humanité et la gloire de Yhwh (1,26-28).
[39] Noter que les faces et les ailes forment une grande inclusion entre les versets 1,6 et 1,11 et que si le verset 7 détaille les pieds, les plantes puis le 8 , les mains (et les côtés ?), le verset 9 revient sur les ailes en les associant à la face comme direction tandis que le verset 10 détaille les quatre faces. La caractérisation première de ces créatures hybrides est le(s) visage(s) et les ailes, manifestement au-delà donc de tout anthropomorphisme.

[40] Anthonioz (en préparation). 
arc et manifestent bien la diversité plutôt que la systématisation de la réflexion sapientiale [41]. Ce discours de la sagesse reflète aussi un certain cosmopolitisme comme la richesse et la diversité religieuse de l'époque hellénistique. Si les influences gréco-égyptiennes font surface ici ou là, elles n'épuisent pas non plus les représentations de la figure de la sagesse. Celle-ci en est d'autant plus cosmopolite [42].

Le texte source de la Sagesse personnifiée est sans conteste $\operatorname{Pr} 1-9$. L'éloge qui se développe en $\operatorname{Pr} 8$ se concentre sur les origines de Sagesse. Cette section est particulièrement importante puisqu'elle pose la question de l'essence de Sagesse, de sa relation à la divinité Yhwh, de sa possible divinisation, voire hypostase dans la réception chrétienne, comme principe premier et personne trinitaire [43]. Mais Sagesse est « créée » par Yhwh (יהוה קנני, 8,22 cf. Si 1,4), littéralement « en tête / (au) début de son chemin » (דרכו ראשית), « avant ses œuvres d'antan » (קדם מפעליו מאז, 8,22). Le sens de procréation du verbe qānâ peut être privilégié, puisqu'il est confirmé par la racine *hwl, répétée deux fois : elle a été « enfantée », quand les abîmes n'étaient pas $(8,24)$. Avant que les montagnes n'aient été enfoncées (הטבעו) [44], avant les collines, elle a été « enfantée » (45], avant que la terre ne soit faite $(8,26)$. Sagesse appartient donc à Yhwh en vertu du fait qu'il l'a créée et enfantée. Il faut souligner la dimension à la fois paternelle et maternelle de la divinité Yhwh dans l'acte de génération : la triple répétition des verbes de création, d'enfantement et d'accouchement ( ${ }^{*} h w l$ ) montre que Sagesse a une origine divine, à l'image des générations divines que l'on peut étudier dans de nombreuses sources anciennes. En ce sens, sa nature peut être comprise comme divine. Aussi est-elle présente à I'œuvre de création et dans cette œuvre de création, Sagesse est auprès de Yhwh (ואהיה אצלו), 'āmôn (אמון) [46], (faisant ses) délices et ceux de
I'humain. À y regarder de près, les résonances philosophiques apparaissent nombreuses et augmentées dans la traduction grecque de la Septante. Dans le Timée, en effet, l'âme du monde et le monde lui-même sont engendrés ainsi que le ciel, le temps et les astres (34b ; 37a.c). Et I'harmonie leur est commune ( $\operatorname{Pr} 8,30$ // Tim 35a ; 36e ; 37a), comme la joie du créateur contemplant son œuvre ( $\operatorname{Pr} 8,31 / / \operatorname{Tim} 37 c)$. Le texte grec aurait ainsi infléchi le sens hébreu [47]. Mais, au-delà des influences philosophiques, la dimension féminine est particulièrement importante à un moment où la divinité s'affirme de manière exclusive et masculine. On peut penser qu'il y a là un correctif théologique. Mais il faut aussi ne pas oublier la dimension pédagogique manifestement féminine : c'est elle qui invite le sage à emprunter ses chemins et à l'embrasser. Si on met en regard les cas où la féminité des déesses est un outil de la polémique, on peut souligner la différence de Dame Sagesse. Sa privation de culte n'est certainement pas anodine pour la place qu'elle occupe auprès de Yhwh : proche de Yhwh, elle partage sa nature divine, sur un mode philosophique que l'on connaît dans le monde grec. Elle ne risque donc pas de lui faire d'ombre sur le plan cultuel.

Ainsi, les quatre cas étudiés dans cette deuxième partie montrent que les attributions de genre répondent à des enjeux propres selon les textes. Si elles participent encore et toujours de la polémique idéologique biblique, elles invitent pourtant à la dépasser pour dire et décrire le caractère insaisissable du divin, qui est exprimé à la fois par une certaine indifférence au genre, dans l'association des genres, ou dans la complémentarité des genres. Dame Sagesse reste un cas à part qui démontre que, même à une époque tardive, la notion d'une divinité masculine et exclusive pouvait être encore débattue ou du moins nuancée.
[41] Les cas avérés sont finalement peu nombreux. Les textes comme Jb 28, Ba 3,9-4,4, ou les manuscrits de Qumrân, doivent manifestement sortir du corpus de la sagesse personnifiée. Et même pour les témoins comme le Siracide ou le livre de la Sagesse, la notion de personnification doit être maniée avec prudence. Des influences textuelles ne s'en font pas moins jour de Job à Baruch, du Siracide au livre de la Sagesse, de Baruch à Hénoch et très souvent à la source de ces influences se tient le témoin des Proverbes.

[42] Voir les travaux réunis dans ANTHonioz \& Dogniez 2021.

[43] Concernant les hypothèses de la divinisation de Dame
Sagesse, voir entre autres LANG 1995, p. 61-98 ; BONNARD 1979, p. 117-149; KAYATZ 1966.

[44] Voir Jb 38,6.

[45] Voir aussi Ps 51,7 («Voici, j'ai été enfanté dans I'iniquité, et dans le péché ma mère m'a conçu »).

[46] Au sujet de ce terme, une littérature abondante existe. Deux interprétations majeures ont été proposées selon I'analyse grammaticale et la re-vocalisation du terme, soit « enfant » (āmûn), soit « artisan » (en relation avec l'akkadien ummânu). Voir, par exemple, HuRowitz 1999, p. 391-400 ; CAZELLES 1999, p. 45-55.

[47] HAMONVILLE 2000, p. 110-112. Sur la culture grecque du traducteur voir de manière plus large p. 102-108. 


\section{CONCLUSION}

Les différents cas analysés, qu'il s'agisse des dénominations divines ou du corps divin, montrent qu'en matière de représentations, la question du genre est essentielle mais loin d'être tranchée dans les textes bibliques : les cas mis en évidence sont au moins trois : 1) des dénominations et des représentations sans attribution de genre clairement distincte, 2) I'association étroite des genres et 3) la fluidité du genre. Les textes bibliques deviennent par là des témoins précieux en regard des sources contemporaines, épigraphiques et matérielles : ils démontrent que la notion de genre est particulièrement féconde pour penser le divin et les représentations divines, au-delà de la sexuation des dieux et au-delà des contextes d'écriture souvent idéologiques et polémiques dans la Bible hébraïque. La question du genre peut-être plus que toute autre rend compte non seulement des traditions et de leur « repensement » mais de leur grande fluidité.

\section{ABRÉVIATIONS}

CAD : Chicago Assyrian Dictionary

SAA : State Archives of Assyria

\section{BIBLIOGRAPHIE}

ACKerman, Susan, 1999, «"And the Women Knead Dough": The Worship of the Queen of Heaven in Sixth-Century Judah », dans Alice Bach (éd.), Women in the Hebrew Bible, London, p. 21-32.

Anthonioz, Stéphanie, 2014, « Astarte in the Bible and her Relation to Ashera », dans David T. Sugimoto (éd.), Ishtar / Astarte / Aphrodite: Transformation of a Goddess, Fribourg - Göttingen, p. 125-139.

Anthonroz, Stéphanie, en préparation, «Chérubins / Keruvim : des comparatismes oubliés », dans Philippe Abrahami, Stéphanie Anthonioz (éd.), Les Chérubins / Keruvim, Münster.

Anthonioz, Stéphanie \& Dogniez, Cécile, 2021, Représentations et personnification de la sagesse dans l'Antiquité et au-delà, Paris (Orient et Méditerranée).

Anthonioz, Stéphanie \& Tenaillon, Nicolas, 2017, «La personnification de la sagesse : Regards croisés philosophique et biblique », Judaïsme ancien - Ancient Judaism 5, p. 77-108.

Archr, Alfonso, 1993, « Divinités sémitiques et divinités de substrat : Le cas d'Išhara et d'Ištar à Ébla », dans Jean-Claude Margueron \& Jean-Marie Durand (dir ;), Mari : Annales de recherches interdisciplinaires 7, p. 71-78.

Biale, David, 1982, « The God with Breasts: El Shaddai in the Bible », History of Religions 21, p. 240-256.

Binger, Tilde, 1995, « Ashera in Israel », Scandinavian Journal of the Old Testament 9, p. 3-18.

BlAžEK, Václav, 1996, « The Semitic Divine Name *'attar(-at-) and its Possible Afroasiatic Cognates », dans Petr Zemánek (éd.), Studies in Near Eastern Languages and Literatures: Memorial Volume for Karel Petráček, Praha, p. 133-141.

Bonnard, Pierre-Émile, 1979, « De la Sagesse personnifiée dans I'Ancien Testament à la Sagesse en personne dans le Nouveau », dans Maurice Gilbert (éd.), La Sagesse de l'Ancien Testament, Leuven, p. 117-149.

Bonnet, Corinne, 1996, Astarté : dossier documentaire et perspectives historiques, Roma (Collezione di studi fenici 37).

Bonnet, Corinne \& Niehr, Herbert, 2014, La religion des Phéniciens et des Araméens, Genève.

BordreuIL, Pierre, 1998, «Ashtart de Mari et les dieux d'Ougarit », Mari : Annales de recherches interdisciplinaires 4, Actes du colloque international du CNRS 620, Strasbourg, 29-30 juin, 1er juillet 1983, p. 545-547.

Bron, François, 1999-2000, « Divinités communes à la Syrie-Palestine et à l'Arabie du Sud préislamique », Aula Orientalis 17-18, p. 437-440.

Canney, Maurice A., 1922, « Shaddai », Expository Times 34, p. 332.

CaZelles, Henri, 1999, «Ahiqar, Ummân and Amun, and Biblical Wisdom Texts », dans Ziony Zevit et al. (éd.), Solving Riddles and Untying Knots: Biblical, Epigraphic, and Semitic Studies in Honor of Jonas C. Greenfield, Winona Lake, p. 45-55.

CoRnelius, Izak, 1997, « The Many Faces of God: Divine Images and Symbols in Ancient Near Eastern Religions », dans Karel van der Toorn (éd.), The Image and the Book, Leuven, p. 21-43.

Cornelius, Izak, 2004, The Many Faces of the Goddess: The Iconography of the Syro-Palestinian Goddesses Anat, Astarte, Qedeshet, and Asherah c. 1500-1000 BCE, Fribourg -Göttingen. 
Cross, Frank M., 1973, Canaanite Myth and Hebrew Epic: Essays in the History of the Religion of Israel, Cambridge.

DAY, John, 1986, «Asherah in the Hebrew Bible and Northwest Semitic Literature », Journal of Biblical Literature 105, p. 385-408.

Delcor, Mathias, 1982, « Le culte de la "Reine du Ciel" selon Jer 7,18; 44,17-19,25 et ses survivances : aspects de la religion populaire féminine aux alentours de l'Exil en Juda et dans les communautés juives d'Égypte », dans Wilhelmus C. Delsman et al. (éd.), Von Kanaan bis Kerala, Kevelaer - Neukirchen-Vluyn, p. 101-122.

Dhorme, Édouard, 1923, L'emploi métaphorique des noms de parties du corps en hébreu et en akkadien, Paris.

Hamonville, David-Marc d', 2000, Les Proverbes / trad. du texte grec de la Septante, introd. et notes, Paris (LXX 17).

Hensel, Benedikt, 2020, «Yahwistic Diversity and the Hebrew Bible: State of the Field, Desiderata and Research Perspectives in a Necessary Debate on the Formative Period of Judaism(s) », dans Benedikt Hensel et al. (éd.), Yahwistic Diversity and the Hebrew Bible: Tracing Perspectives of Group Identity from Judah, Samaria, and the Diaspora in Biblical Traditions, Tübingen.

HeSS, Richard S., 1996, « Asherah or Asherata », Orientalia 65, p. 209-219.

Hulster, Izaak J. de, 2017, Figurines in Achaemenid Period Yehud. Jerusalem's History of Religion and Coroplastics in the Monotheism Debate / Figurinen im achämenidischen Jehud: Die Religionsgeschichte Jerusalems und Koroplastik in der Monotheismus-Debatte, Tübingen.

Hurowitz, Victor A., 1999, « Nursling, Advisor, Architect? אמון and the Role of Wisdom in Proverbs 8:22-31 », Biblica 80, p. 391-400.

Kayatz, Christa, 1966, Studien zu Proverbien 1-9: Eine form- und motivgeschichtliche Untersuchung unter Einbeziehung ägyptischen Vergleichsmaterials, Neukirchen-Vluyn (Wissenschaftliche Monographien zum Alten und Neuen Testament 22).

LANG, Bernhard, 1995, « Figure ancienne, figure nouvelle de la sagesse en $\operatorname{Pr} 1$ à 9 », dans Jacques Trublet (éd.), La sagesse biblique de I'Ancien au Nouveau Testament, Paris, p. 61-98.

LuтzKy, Harriet, 1998, « Shadday as a Goddess Epithet », Vetus Testamentum 48, p. 15-36.

MARGAlit, Baruch, 1990, « The Meaning and Significance of Asherah », Vetus Testamentum 40, p. 264-297.

Müller, Hans-Peter, 2001, «שתרת, 'aštôret », Theological Dictionary of the Old Testament 1, p. 423-434.

Olyan, Saul M., 1987, « Some Observations Concerning the Identity of the Queen of Heaven », Ugarit-Forschungen 19, p. 161-174.

Parpola, Simo \& Watanabe, Kazuko, 1988, Neo-Assyrian Treaties and Loyalty Oaths, Helsinki (State Archives of Assyria 2). Pettey, Richard J., 1990, Asherah: Goddess of Israel, New York.

Pongratz-Leisten, Beate, 2011, « Divine Agency and Astralization of the Gods in Ancient Mesopotamia », dans Beate Pongratz-Leisten (éd.), Reconsidering the Concept of Revolutionary Monotheism, Winona Lake, p. 137-187.

Richelle, Matthieu, 2018, « Arbres sacrés, poteaux cultuels et déesse Ashéra dans I'Israël ancien », dans Pierre-Sylvain Filliozat (éd.), L'arbre en Asie, Actes du colloque de la Société Asiatique 2016, Paris, p. 95-119.

SмIтн, Mark S., 1995, « The God Athtar in the Ancient Near East and His Place in KTU 1.6 I », dans Ziony Zevit et al. (éd.), Solving Riddles and Untying Knots, Winona Lake, p. 627-640.

Sommer, Benjamin D., 2009, The Bodies of God and the World of Ancient Israel, Cambridge.

Tномаs, Ryan, 2016, « The Identity of the Standing Figures on Pithos A from Kuntillet 'Ajrud: A Reassessment », Journal of Ancient Near Eastern Religions 16, p. 121-191.

ThомAS, Ryan, 2017, « The Meaning of Asherah in Hebrew Inscriptions », Semitica 59, p. 157-218.

VAN Der Toorn, Karel, 1992, «Anat-Yahu, Some Other Deities, and the Jews of Elephantine», Numen 39, p. 80-101.

VAN DER Toorn, Karel, 2016, «Eshem-Bethel and Herem-Bethel: New Evidence from Amherst Papyrus 63 », Zeitschrift für die alttestamentliche Wissenschaft 128, p. 668-680.

Vriezen, Karel J. H., 1996, « Cakes and Figurines: Related Women's Cultic Offerings in Ancient Israel », dans Bob Becking et al. (éd.), On Reading Prophetic Texts: Gender-specific and related studies in memory of Fokkelien van Dijk-Hemmes, Leiden, p. 251-264.

Vriezen, Karel J. H., 2001, « Archaeological Traces of Cult in Ancient Israel », dans Bob Becking et al. (éd.), Only One God? Monotheism in Ancient Israel and the Veneration of the Goddess Asherah, Sheffield, p. 45-80.

Westenholz, Joan G., 2012, « The Body and the Mind in Mesopotamian Traditions », dans Angelika Berlejung et al. (éd.), Menschenbilder und Körperkonzepte im Alten Israel, in Ägypten und im Alten Orient, Tübingen, p. 459-477.

Wiggins, Steve A., 2003, «Pidray, Tallay and Arsay in the Baal Cycle», Journal of Northwest Semitic Languages 29, p. 83-101. WrLson-Wright, Aren M., 2016, Athtart: The Transmission and Transformation of a Goddess in the Late Bronze Age, Tübingen. Wilson-Wright, Aren M., 2019, «The Helpful God: A Reevaluation of the Etymology and Character of ('êl) šadday », Vetus Testamentum 69, p. 149-166.

Zernecke, Anna E., 2013, «The Lady of the Titles: The Lady of Byblos and the Search for her "True Name" », Die Welt des Orients 43, p. 227-242. 